\title{
Different clinical patterns of IgG4-RD patients with and without eosinophilia
}

\author{
Xia Zhang ${ }^{1}$, Panpan Zhang ${ }^{1}$, Jieqiong $\mathrm{Li}^{1}$, Yujie He${ }^{2}$, Yunyun Fei ${ }^{1}$, Linyi Peng ${ }^{1}$, Qun Shi ${ }^{1}$, \\ Wen Zhang ${ }^{1 *}$ \& Yan Zhao ${ }^{1^{*}}$
}

It has been reported that patients with IgG4-related disease (IgG4-RD) showed an elevated incidence of eosinophilia. We aim to explore the clinical patterns of IgG4-RD patients with and without eosinophilia. Four hundred and twenty-five IgG4-RD patients referred to Peking Union Medical College Hospital were enrolled. Blood eosinophil count higher than $0.5 \times 10^{9} / \mathrm{L}$ was defined as eosinophilia. Clinical features of all the participants were collected and analyzed statistically. Eighty-seven patients (20\%) with eosinophilia were found. As compared to those with a normal range of blood eosinophil count, male predominance, longer disease duration, increased prevalence of dacryoadenitis, sialadenitis, lymphadenopathy, and skin rash, higher IgG4-RD responder index, more organ involvement and higher levels of serum Ig $4(17.0 \mathrm{~g} / \mathrm{L}$ vs $6.5 \mathrm{~g} / \mathrm{L}, P<0.001)$ was found in patients with eosinophilia. There was no significant difference in the incidence of allergic disease between the two groups. Peripheral eosinophil counts were positively correlated with disease duration, the number of involved organs, IgG4-RD responder index, and serum IgG4. Higher recurrence rate during follow-up period was found in patients with eosinophilia [28.6\% (20/70) vs $17.1 \%(42 / 245), P=0.034]$. IgG4-RD patients with eosinophilia exhibited different clinical patterns from patients without. Eosinophilia appeared independent of allergies in IgG4-RD.

Eosinophils are terminally differentiated cells of the myeloid lineage implicated in the pathogenesis of numerous inflammatory processes ${ }^{1}$. In response to a variety of stimuli, mature peripheral blood eosinophils are recruited into the tissue, where they modulate immune responses through multiple mechanisms. Eosinophils secrete a series of cytokines capable of promoting T cell expansion, and T helper type 1 (Th1)/2 (Th2) polarization ${ }^{2}$. Eosinophilia is defined as an elevation of eosinophils in the bloodstream. Many diseases are associated with eosinophilia, including parasitic diseases, allergy, autoimmune diseases, malignancy, primary hypereosinophilic syndrome . $^{3}$

Immunoglobulin G4-related disease (IgG4-RD) is an increasingly recognized chronic fibroinflammatory disorder with multiple organ involvement, including salivary glands, lacrimal glands, pancreas, retroperitoneum, kidneys, lymph nodes, lungs, and liver among others. IgG4-RD is pathologically characterized by IgG4-positive lymphoplasmacytic infiltration, storiform fibrosis and obliterative phlebitis ${ }^{4}$. The pathogenesis of IgG4-RD remains poorly understood.

Recently, eosinophilia had been reported to be associated with IgG4-RD to varying degrees (11-38\% $)^{5-11}$. This eosinophilia appeared inherent to the IgG4-RD rather than atopic disease ${ }^{10,11}$. Some patients with high eosinophils as the first manifestation were finally confirmed to be IgG4-RD ${ }^{12-14}$. Eosinophils also infiltrate in the involved tissues, which is generally mild to moderate but can be remarkable in some cases ${ }^{15}$. Eosinophilic angiocentric fibrosis was recently described as a form of IgG4-related systemic disease ${ }^{16}$. In 2010, Sah et al. reported a similar clinical profile in type 1 autoimmune pancreatitis patients with and without peripheral eosinophilia ${ }^{11}$. In 2014, Della-Torre et al. reported that there was a positive correlation between eosinophil count and serum $\operatorname{IgG} 4^{10}$. In 2017, Culver et al. revealed that eosinophil count was positively correlated with both serum $\operatorname{IgE}$ and serum IgG4. However, there was no statistical difference in serum IgG4 levels between IgG4-RD patients with and without eosinophilia ${ }^{7}$.

\footnotetext{
${ }^{1}$ Department of Rheumatology, Peking Union Medical College Hospital, Chinese Academy of Medical Science \& Peking Union Medical College, Key Laboratory of Rheumatology and Clinical Immunology, Ministry of Education, Beijing, China. ${ }^{2}$ Department of Rheumatology, First Affiliated Hospital of Zhengzhou University, Zhengzhou, China. *email: zhangwen91@sina.com; zhaoyan_pumch2002@aliyun.com
} 


\begin{tabular}{|c|c|c|c|c|}
\hline & Total $(n=425)$ & $\begin{array}{l}\text { Patients without } \\
\text { eosinophilia }(n=338)\end{array}$ & $\begin{array}{l}\text { Patients with } \\
\text { eosinophilia }(n=87)\end{array}$ & Pvalue \\
\hline Peripheral blood eosinophil count (cells per $\mu \mathrm{L}$ ) & $210(100-420)$ & $170(80-260)$ & $770(610-1100)$ & $<0.001$ \\
\hline Age (years) $)^{\#}$ & $54 \pm 13$ & $54 \pm 13$ & $53 \pm 15$ & 0.442 \\
\hline Male (\%) & $60.4 \%$ & $57.1 \%$ & $73.3 \%$ & 0.007 \\
\hline Disease duration (months) $^{*}$ & $12(4-36)$ & $10(4-36)$ & $12(6-48)$ & 0.034 \\
\hline Allergy history & $49.2 \%$ & $47.6 \%$ & $55.2 \%$ & 0.145 \\
\hline IgG4-RD RI* & $12(7-16)$ & $11(7-15)$ & $13(10-17)$ & $<0.001$ \\
\hline Numbers of organs involved & $3.31 \pm 1.71$ & $3.17 \pm 1.63$ & $3.86 \pm 1.89$ & 0.003 \\
\hline HBG $(\mathrm{g} / \mathrm{L})^{\#}$ & $134.2 \pm 18.3$ & $134.2 \pm 18.1$ & $134.3 \pm 19.2$ & 0.687 \\
\hline PLT $(\times 109 / \mathrm{L})^{*}$ & $231(195-280)$ & $231(191-281)$ & $237(206-278)$ & 0.254 \\
\hline $\mathrm{WBC}\left(\times 10^{9} / \mathrm{L}\right)^{*}$ & $6.7(5.6-8.0)$ & $6.5(5.5-7.7)$ & $7.32(5.90-9.03)$ & $<0.001$ \\
\hline $\operatorname{ESR}(\mathrm{mm} / \mathrm{h})^{*}$ & $18(8-51)$ & $17(8-44)$ & $34(12-76)$ & 0.002 \\
\hline $\mathrm{CRP}(\mathrm{mg} / \mathrm{L})^{*}$ & $2.1(0.8-7.6)$ & $2.3(0.7-7.6)$ & $2.1(1.3-7.4)$ & 0.342 \\
\hline $\operatorname{IgG}(\mathrm{g} / \mathrm{L})^{*}$ & $18.7(14.6-24.7)$ & $18.1(14.2-23.3)$ & $24.1(16.4-32.6)$ & $<0.001$ \\
\hline $\operatorname{IgA}(\mathrm{g} / \mathrm{L})^{*}$ & $2.06(1.39-2.75)$ & $2.14(1.54-2.85)$ & $1.54(0.96-2.13)$ & $<0.001$ \\
\hline $\operatorname{IgM}(\mathrm{g} / \mathrm{L})^{*}$ & $0.77(0.55-1.22)$ & $0.81(0.57-1.24)$ & $0.70(0.48-1.05)$ & 0.094 \\
\hline $\operatorname{IgG1}(\mathrm{g} / \mathrm{L})^{*}$ & $9.14(7.45-11.00)$ & $9.06(7.30-10.60)$ & $9.68(7.65-13.10)$ & 0.009 \\
\hline $\operatorname{IgG} 2(\mathrm{~g} / \mathrm{L})^{*}$ & $5.73(4.42-7.58)$ & $5.88(4.56-7.64)$ & $5.27(3.75-7.18)$ & 0.022 \\
\hline $\operatorname{IgG} 3(\mathrm{~g} / \mathrm{L})^{*}$ & $0.45(0.24-0.86)$ & $0.42(0.23-0.81)$ & $0.55(0.31-0.98)$ & 0.012 \\
\hline $\operatorname{IgG} 4(\mathrm{~g} / \mathrm{L})^{*}$ & $7.72(3.18-17.50)$ & $6.50(2.74-14.38)$ & $17.00(5.60-31.55)$ & $<0.001$ \\
\hline IgG4/IgG ${ }^{*}$ & $0.34(0.17-0.53)$ & $0.31(0.16-0.48)$ & $0.49(0.25-0.61)$ & $<0.001$ \\
\hline $\operatorname{IgE}(\mathrm{KU} / \mathrm{L})^{*}$ & 347 (126-752) & $302(119-662)$ & 480 (157-1164) & 0.010 \\
\hline $\mathrm{C} 3(\mathrm{~g} / \mathrm{L})^{*}$ & $0.94(0.75-1.11)$ & $0.96(0.79-1.11)$ & $0.79(0.58-1.15)$ & 0.088 \\
\hline $\mathrm{C} 4(\mathrm{~g} / \mathrm{L})^{*}$ & $0.17(0.12-0.24)$ & $0.18(0.13-0.24)$ & $0.12(0.05-0.19)$ & $<0.001$ \\
\hline
\end{tabular}

Table 1. Baseline demographic and clinical characteristics of patients with IgG4-RD. HGB, hemoglobin; PLT, platelet cell; WBC, white blood cell; GC mono, glucocorticoids monotherapy; GC \& IM, glucocorticoids combined immunosuppressants therapy; IM mono, immunosuppressants monotherapy; TCM, traditional Chinese medicine; *Values are median (IQR); ${ }^{*}$ Values are Mean \pm Standard Deviation.

In this study, we investigated the prevalence of eosinophilia in IgG4-RD patients in the largest prospective IgG4-RD cohort in China, and report here for the first time that patients with eosinophilia presented with significantly different clinical patterns in comparison to those with normal peripheral eosinophil count.

Results

Patients with eosinophilia showed male predominance and longer disease duration. Among 425 patients in this cohort, the median blood eosinophil count was 210 cells per $\mu \mathrm{L}$ (IQR 100-420; Table 1). Eighty-seven patients (20\%) showed peripheral blood eosinophil count higher than 500 per $\mu \mathrm{L}\left(0.5 \times 10^{9}\right.$ per $\left.\mathrm{L}\right)$, 134 patients (32\%) showed peripheral blood eosinophil ratio more than $5 \%$. The eosinophil count higher than 1500 cells per $\mu \mathrm{L}$ were recorded in $13(3 \%)$ patients.

Patients with eosinophilia were more likely to be male $(73.3 \%$ vs. $57.1 \%, P=0.007)$ and with longer disease duration [12(6-48) vs. $10(4-36), P=0.034]$ (Table 1$)$.

Patients with eosinophilia exhibited more organ involvement and higher serum IgG4 level. Patients with eosinophilia had higher incidence of dacryoadenitis $(61.9 \%$ vs $16.4 \%, P=0.014)$, submandibular sialadenitis ( $65.9 \%$ vs $49.5 \%, P=0.007)$, lymphadenopathy $(55.3 \%$ vs $42.0 \%, P=0.037)$ and skin rash $(7.4 \%$ vs $2.7 \%, P=0.034)$ (Fig. $1 \mathrm{~A})$, as well as higher IgG4-RD RI and more organ involvement $(P<0.05$, Table 1). Interestingly, there was no significant difference in the incidence of allergic diseases between patients with and without eosinophilia (Table 1). In the laboratory inspection, patients with eosinophilia showed higher peripheral WBC, ESR, serum IgG, IgG1, IgG3, IgG4, IgE, and IgG4/IgG ratio, while lower serum $\operatorname{IgA}, \operatorname{IgG} 2$ and C4 level $(P<0.05$, Table 1).

Peripheral eosinophil counts correlated to disease burden in IgG4-RD patients. Correlation analysis revealed that peripheral eosinophil counts were positively correlated with disease duration, numbers of organs involved, and IgG4-RD RI (Table 2). Besides, blood eosinophil counts were also positively related to peripheral WBC, ESR, IgG, IgG1, IgG3, IgG4, IgE, and IgG4/IgG ratio, whereas negatively correlated to IgA, IgM, IgG2 and C4, as showed in Table 2. All these indicate that peripheral eosinophil counts were associated with disease burden in IgG4-RD patients.

Patients with eosinophilia were more liable to undergo disease relapse. In the overall 425 patients, 46 patients $(10.8 \%)$ were untreated and under observation, 110 patients $(25.9 \%)$ received glucocorticoids monotherapy (GC mono), 60 patients (14.1\%) received immunosuppressants monotherapy (IM mono), 

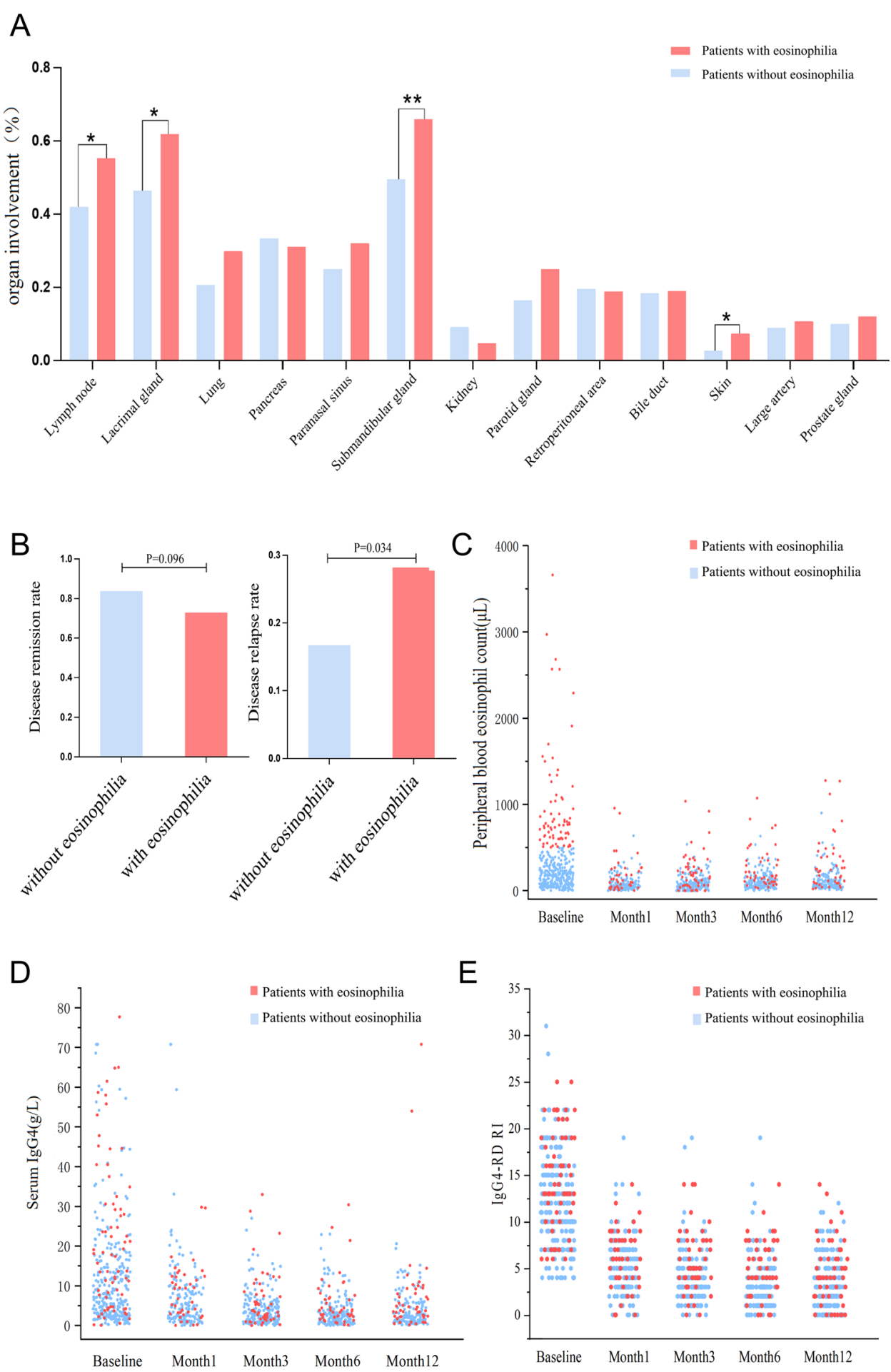

Figure 1. Organs involvement and outcome of patients with and without eosinophilia. (A) Organ involvement of the two groups of patients. (B) Disease remission rate and recurrence rate. (C-E) Changes of peripheral blood eosinophil count, serum IgG4, IgG4-RI of the two groups of patients during follow-up. IgG4-RD RI, IgG4-RD responder index. $* P<0.05 ; * P<0.01$.

208 patients (48.9\%) received combination therapy with GC and IM, 1 patient $(0.2 \%)$ chose traditional Chinese medicine treatment. Treatment options displayed no significant difference between the two groups.

Remission rates were evaluated six months after initiating remission induction treatment with GC or GC combined IM. It seemed that patients with eosinophilia tend to have a lower remission rate, but the difference was not statistically significant [83.8\% (160/191) vs. 72.9\% (35/48), $P=0.096]$ (Fig. 1B). IgG4-RD RI dropped by half or more at the $6^{\text {th }}$ - month follow-up in the two groups were $92.2 \%(202 / 219)$ and $89.7 \%(52 / 58)$, respectively $(P=0.526)$. 


\begin{tabular}{|l|c|c|}
\hline \multirow{2}{*}{ Parameters } & \multicolumn{2}{|l|}{ Peripheral blood eosinophil count } \\
\cline { 2 - 3 } & Spearman $\mathbf{r}$ & P value \\
\hline Age (years) & 0.005 & 0.922 \\
\hline Disease duration (months) & 0.140 & 0.006 \\
\hline Numbers of organs involved & 0.153 & 0.002 \\
\hline IgG4-RD RI & 0.169 & 0.001 \\
\hline WBC $\left(\times 10^{9} / \mathrm{L}\right)$ & 0.190 & $<0.001$ \\
\hline ESR $(\mathrm{mm} / \mathrm{h})$ & 0.104 & 0.040 \\
\hline CRP $(\mathrm{mg} / \mathrm{L})$ & 0.012 & 0.827 \\
\hline IgG $(\mathrm{g} / \mathrm{L})$ & 0.240 & $<0.001$ \\
\hline IgA $(\mathrm{g} / \mathrm{L})$ & -0.244 & $<0.001$ \\
\hline IgM $(\mathrm{g} / \mathrm{L})$ & -0.128 & 0.010 \\
\hline IgG1 $(\mathrm{g} / \mathrm{L})$ & 0.102 & 0.042 \\
\hline IgG2 $(\mathrm{g} / \mathrm{L})$ & -0.108 & 0.030 \\
\hline IgG3 $(\mathrm{g} / \mathrm{L})$ & 0.115 & 0.021 \\
\hline IgG4 $(\mathrm{g} / \mathrm{L})$ & 0.335 & $<0.001$ \\
\hline IgG4/IgG & 0.320 & $<0.001$ \\
\hline IgE $(\mathrm{KU} / \mathrm{L})$ & 0.179 & 0.002 \\
\hline C3 $(\mathrm{g} / \mathrm{L})$ & -0.106 & 0.094 \\
\hline C4 $(\mathrm{g} / \mathrm{L})$ & -0.200 & 0.002 \\
\hline & & \\
\hline
\end{tabular}

Table 2. Correlations between peripheral blood eosinophil count and clinical indicators.

\begin{tabular}{|c|c|c|c|}
\hline & $\begin{array}{l}\text { Patients without } \\
\text { eosinophilia }\end{array}$ & $\begin{array}{l}\text { Patients with } \\
\text { eosinophilia }\end{array}$ & P value \\
\hline \multicolumn{4}{|l|}{ Serum IgG4 at baseline } \\
\hline $\operatorname{Normal}(<1.35 \mathrm{~g} / \mathrm{L})$ & $4.7 \%(16 / 338)$ & $3.4 \%(3 / 87)$ & 0.625 \\
\hline \multicolumn{4}{|c|}{ Serum IgG4 at $1^{\text {st }}$ month follow-up } \\
\hline $\operatorname{Normal}(<1.35 \mathrm{~g} / \mathrm{L})$ & $17.7 \%(29 / 164)$ & $13.5 \%(5 / 37)$ & 0.430 \\
\hline Decrease by $50 \%$ or more* & $47 \%(77 / 164)$ & $54.1 \%(20 / 37)$ & 0.626 \\
\hline \multicolumn{4}{|c|}{ Serum IgG4 at $3^{\text {rd }}$ month follow-up } \\
\hline $\operatorname{Normal}(<1.35 \mathrm{~g} / \mathrm{L})$ & $34.7 \%(77 / 222)$ & $15.8 \%(9 / 57)$ & 0.003 \\
\hline Decrease by $50 \%$ or more* & $72.5 \%(161 / 222)$ & $80.7 \%(46 / 57)$ & 0.317 \\
\hline \multicolumn{4}{|c|}{ Serum IgG4 at $6^{\text {th }}$ month follow-up } \\
\hline $\operatorname{Normal}(<1.35 \mathrm{~g} / \mathrm{L})$ & $40.2 \%(78 / 194)$ & $14.6 \%(7 / 48)$ & 0.001 \\
\hline Decrease by $50 \%$ or more* & $70.1 \%(136 / 194)$ & $81.3 \%(39 / 48)$ & 0.122 \\
\hline \multicolumn{4}{|c|}{ Serum IgG4 at $12^{\text {th }}$ month follow-up } \\
\hline Normal $(<1.35 \mathrm{~g} / \mathrm{L})$ & $37.8 \%(65 / 172)$ & $19.1 \%(9 / 47)$ & 0.011 \\
\hline Decrease by $50 \%$ or more* & $73.8 \%(127 / 172)$ & $70.2 \%(33 / 47)$ & 0.620 \\
\hline
\end{tabular}

Table 3. Different levels of serum IgG4 during follow-up. *As compared to serum IgG4 levels at baseline.

Patients were followed up for $25 \pm 15$ months. The disease relapse rate was calculated in 315 patients who were followed up for three months or longer. The final results showed that the total recurrence rate was $19.7 \%(62 / 315)$, and patients with eosinophilia showed a higher relapse rate than patients without eosinophilia $[28.6 \%(20 / 70)$ vs. $17.1 \%(42 / 245), P=0.034$ ] (Fig. 1A). The mean time of recurrence was around 12th (7-24) month, no significant difference was found between two groups [11 (6-24) month vs. $12(7-27)$ month, $P=0.509]$. Patients with hypereosinophilia ( $>1500$ cells per $\mu \mathrm{L}$ ) displayed a trend of higher recurrence rate than patients with blood eosinophil count range from 500 to 1500 cells per $\mu \mathrm{L}$, but the difference was not significant [33.3\% (4/12) vs. 27.6\% (16/58), $P=0.768]$. A lower proportion of patients with eosinophilia had a blood IgG4 level reaching the normal range at the 3rd, 6th, 12th-month follow-up (Table 3).

During the follow-up period, patients with eosinophilia improved significantly, and the peripheral blood eosinophils fell to normal range $(<500 / \mu \mathrm{L})$ in $92.6 \%(50 / 54), 80.9 \%(38 / 47)$ and $83 \%(35 / 42)$ at the 3rd, 6th, 12th-month follow-up, respectively. While three patients without eosinophilia at baseline assessment developed eosinophilia during follow-up, and these patients showed no signs of disease relapse (Fig. 1C). The trends of serum IgG4 and IgG4-RD RI levels during follow-up were shown in Fig. 1D,E. Univariable logistic regression analysis revealed that eosinophilia at baseline and IgA $<0.7 \mathrm{~g} / \mathrm{L}$ (lower limit of the normal range) were two risk factors for sustained eosinophilia at the 12th-month follow-up (Table 4). Serum IgG4 level at 12 months was positively correlated with blood eosinophil count at 12 months $(\mathrm{r}=0.314, P<0.001)$. Comparing patients with different treatment choices, we found that glucocorticoid-based therapy seemed to be more conducive to peripheral blood eosinophil counts declination (Fig. 2). 


\begin{tabular}{|l|l|l|}
\hline \multirow{2}{*}{} & \multicolumn{2}{|l|}{ Eosinophilia at 12 ${ }^{\text {th }}$ month follow-up } \\
\cline { 2 - 3 } & OR $(\mathbf{9 5} \%$ CI) & P value \\
\hline Female sex & $0.18(0.02-1.43)$ & 0.105 \\
\hline Allergy history & $0.86(0.24-3.06)$ & 0.812 \\
\hline Involved organ number & $1.25(0.75-2.10)$ & 0.394 \\
\hline Eosinophilia at baseline & $9.80(2.41-39.81)$ & 0.001 \\
\hline Elevated ESR & $1.43(0.39-5.25)$ & 0.590 \\
\hline Elevated WBC & $3.91(0.93-16.44)$ & 0.063 \\
\hline Elevated IgG & $1.33(0.32-5.51)$ & 0.691 \\
\hline Elevated IgG1 level & $1.24(0.25-6.26)$ & 0.794 \\
\hline Elevated IgG2 level & $0.58(0.14-2.30)$ & 0.434 \\
\hline Elevated IgG3 level & $1.94(0.38-9.92)$ & 0.428 \\
\hline IgA below LLN & $5.79(1.03-32.45)$ & 0.046 \\
\hline Complement below LLN* & $4.65(0.47-46.28)$ & 0.190 \\
\hline
\end{tabular}

Table 4. Univariable logistic regression analysis of risk factors for eosinophilia at $12^{\text {th }}$ month follow-up. *LLN, lower limit of the normal range.

\section{Discussion}

IgG4 related disease (IgG4-RD) is a fibroinflammatory disease with systemic involvement. The pathogenesis of IgG4-RD remains mostly unknown. Many studies have described blood and tissue eosinophilia in patients with IgG4-RD ${ }^{5-11}$. However, most of them didn't describe the comparison between patients with and without eosinophilia. Only one study found that the presence of eosinophilia did not result in a different clinical pattern in patients with type 1 autoimmune pancreatitis ${ }^{11}$. However, in their analysis, they only included patients with single-organ involvement, not systemic IgG4-RD. Hence, the clinical significance of eosinophilia in IgG4-RD is still unclear.

In this study, based on the largest cohort of IgG4-RD patients in China, we report that $20 \%$ of patients had eosinophilia. Patients with and without eosinophilia showed different clinical patterns in terms of organ involvement and laboratory findings. Patients with eosinophilia seemed to have a higher disease burden as indicated by elevated serum IgG, IgG4, ESR, and more organs involved, and higher IgG4-RD RI score. As in an IgG4-RD grouping analysis which reported by Wallace et al. ${ }^{17}$, patients were divided into four groups, Group 4 was male-dominated, characterized by classic Mikulicz syndrome with systemic involvement, showing the highest serum IgG4 levels, but they did not give the comparison of blood eosinophil counts among groups. We previously revealed that systemic IgG4-RD patients with dacryoadenitis and sialadenitis (IgG4-DS) and internal organs involvement had higher serum IgG4 levels and a higher rate of eosinophilia than patients with only IgG4-DS ${ }^{18}$. Patients with only IgG4-DS are more likely to ignore the disease, thus delay the diagnosis and treatment until systemic symptoms occurred, this could be speculated by longer disease duration at diagnosis in patients with eosinophilia. When we independently analyzed the organ involvement number apart from lacrimal glands, salivary glands, and lymph nodes, there was no significant difference between the two groups.

Allergic disorders are common causes of reactive eosinophilia ${ }^{19}$. However, there was no statistically significant difference in the incidence of allergic disease between patients with and without eosinophilia, indicating that eosinophilia is independent of allergies in IgG4-RD patients, which was consistent with the results reported by Della-Torre et al..$^{10}$ and Sah et al. ${ }^{11}$. Wallace et al. ${ }^{20}$ have found that elevated eosinophil count in the baseline was an independent predictor of disease relapse; also, our previous study showed eosinophilia was a risk factor for remission induction failure ${ }^{21}$. Here, we confirmed that IgG4-RD patients with peripheral eosinophilia were more liable to disease relapse.

We found here that peripheral eosinophil counts in IgG4-RD patients were positively correlated with serum IgG4 and disease burden. In addition, serum IgG4 level at 12 months was also positively correlated with the blood eosinophil count at 12 months. IgG4-RD exhibited activated Th2 response ${ }^{22-26}$, and this may be responsible for eosinophilia in IgG4-RD ${ }^{19}$. Otherwise, activated eosinophils release cytokines ${ }^{2}$, which can promote the production of $\operatorname{IgG} 4^{27}$. Although it remains unclear that IgG4 plays a pathogenic or protective role in IgG4-RD ${ }^{28}$, these indicate that eosinophils participate in the pathogenesis of IgG4-RD.

Both CRP and ESR are used to predict inflammatory conditions. ESR level can be affected by factors other than inflammation, such as levels of serum fibrinogen and immunoglobulins. We believe that the difference of ESR levels between the two groups was mainly due to the different distribution of serum IgG and IgG4, as patients with eosinophilia had higher serum IgG, IgG4, while with a similar level of CRP compared to patients with normal eosinophil counts. Patients with eosinophilia showed male predominance, as reported that peripheral blood eosinophil count is skewed according to gender, males usually have higher eosinophil count ${ }^{29,30}$.

Activation of eosinophils led to the production of transforming growth factor (TGF)- $\beta^{2}$, and the latter was reported to participate in the pathogenesis of fibrosis. Fibrosis occurred in the affected organs of advanced IgG4-RD patients. We found that peripheral eosinophil counts in IgG4-RD patients were positively correlated to treatment-free disease duration; patients with eosinophilia showed longer disease duration. These indicate that eosinophilia may occur in the advanced stage of IgG4-RD, and may participate in the fibrosis of affected tissues. 
A
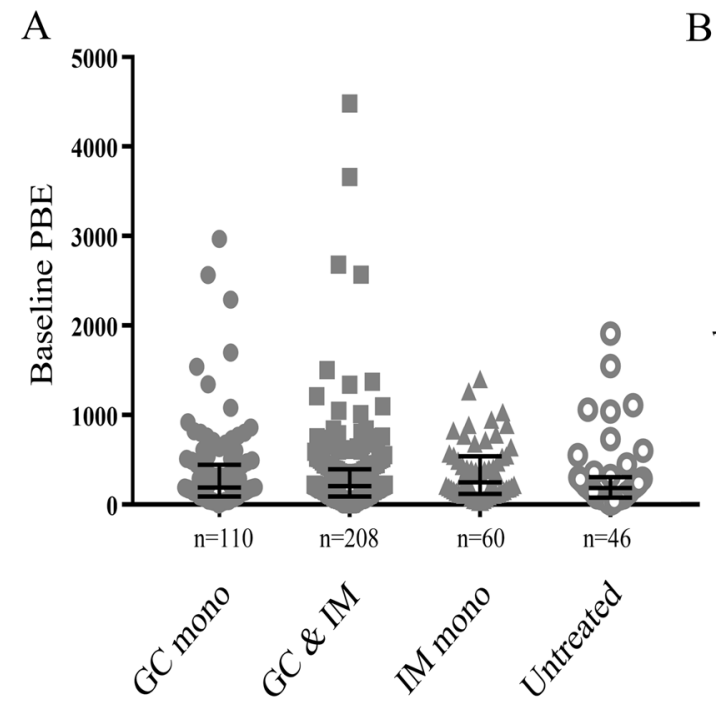

$\mathrm{C}$

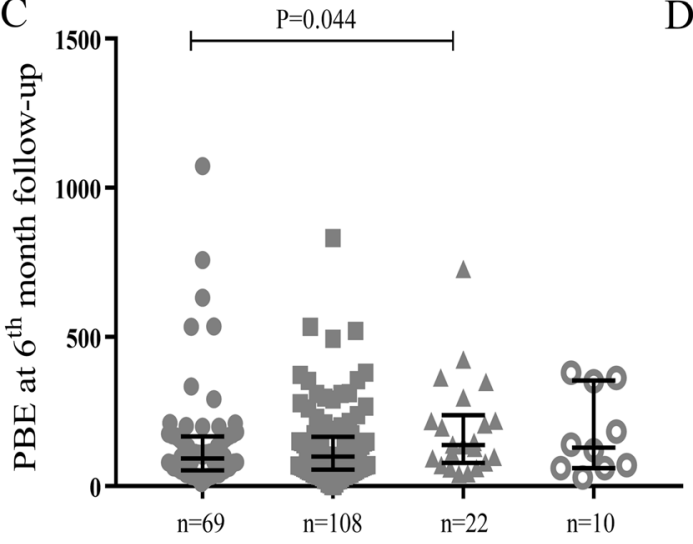

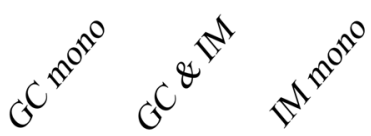

B

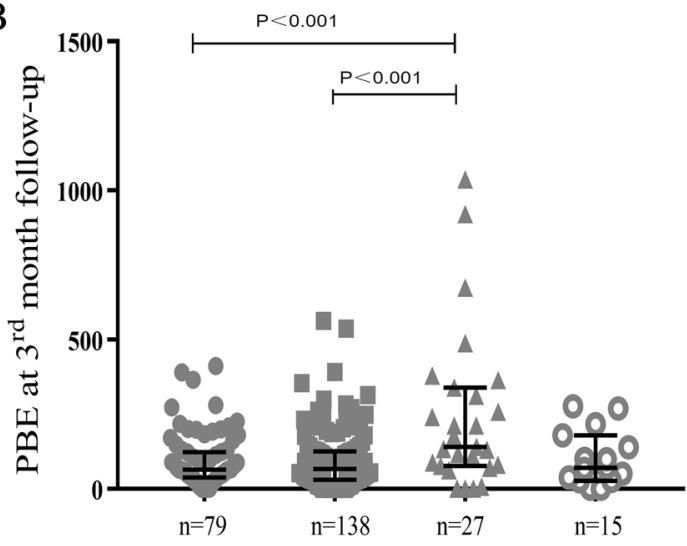

$\mathrm{D}$

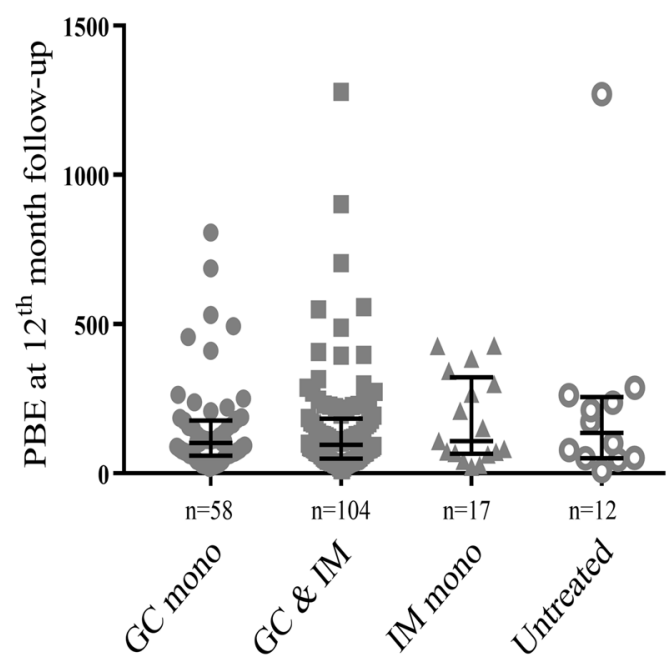

Figure 2. Changes in peripheral blood eosinophil (PBE) counts during follow-up, grouped according to treatment options. (A-D) display peripheral blood eosinophil counts at baseline, the third month follow-up, the sixth-month follow-up, and the 12th month follow-up, respectively. Bars represent median and interquartile ranges. GC mono, glucocorticoids monotherapy; GC \& IM, combination therapy with glucocorticoids and immunosuppressants; IM mono, immunosuppressants monotherapy.

To date, several treatment approaches, including glucocorticoids, myelosuppressive drugs, leukotriene antagonists, tyrosine kinase inhibitors, IFN- $\alpha$, and anti-IL-5 antibodies, have been described for eosinophilia. Although the role of eosinophilia in the pathogenesis of IgG4-RD is debatable, we found that IgG4-RD patients with peripheral eosinophilia tend to be more liable to disease relapse, this raises the possibility of anti-eosinophil therapy such as monoclonal antibody to IL-5 for IgG4-RD patients with recurrent disease.

Our study has some limitations. This is a single-center study, which may cause bias in interpreting results. Only two patients with eosinophilia had done bone marrow biopsy at baseline and showed elevated bone marrow eosinophils. No further tests for the myeloid or lymphocytic variant hypereosinophilic syndrome (HES) had performed. As proposed by Chen et al. ${ }^{31}$, the diagnosis of idiopathic HES need to rule out IgG4-RD, the differential diagnosis between IgG4-RD and myeloid or lymphocytic variant HES need careful consideration of clinical and laboratory manifestation.

In conclusion, peripheral eosinophilia was associated with a different clinical pattern of IgG4-RD. Eosinophil may play an important role in the development of IgG4-RD, especially in patients with longer disease duration and probably pronounced fibrotic lesion. Our findings paved the way for identifying the role of eosinophils in the pathogenesis of IgG4-RD.

\section{Methods}

Patients and study design. A multidisciplinary collaborative prospective cohort study of IgG4-RD and Mimicry patients has been conducted in Peking Union Medical College Hospital (PUMCH, Beijing, China) since January 2011, registered on ClinicalTrials.gov (ID: NCT01670695). Patients were diagnosed with IgG4-RD according to the 2011 comprehensive diagnostic criteria for IgG4-RD ${ }^{32}$. Fifty-one patients who had a history of 
other rheumatic diseases, infectious diseases, or malignancies, which may cause eosinophilia, were excluded. As of August 2018, 425 newly diagnosed IgG4-RD patients were enrolled in this study.

Eosinophilia in the peripheral blood was defined as eosinophil count greater than 500 per $\mu \mathrm{L}\left(0.5 \times 10^{9}\right.$ per $\left.\mathrm{L}\right)$. We divided the patients into two groups according to whether there was eosinophilia. According to the Allergy Definition of the European Society for Allergy and Clinical Immunology ${ }^{33}$ : (1) a history of clinical allergic diseases; (2) a positive skin test or an increase in serum allergen-specific IgE, while satisfying the definition of both as an allergy. Patients with newly diagnosed IgG4-RD were classified as allergic and non-allergic. All patients visited the doctors regularly at 1 st and every 3 to 6 months intervals follow up.

Clinical data and laboratory parameters including complete blood count (CBC), liver and renal function tests, erythrocyte sedimentation rate (ESR), C-reactive protein (CRP), serum immunoglobulin levels, IgG subclasses, and total IgE levels were collected. Organ involvements were defined by physical examination and imaging findings, including computed tomography (CT), or magnetic resonance imaging (MRI), or Positron Emission Tomography/Computed Tomography (PET-CT). Disease activity was assessed by the IgG4-RD responder index $(\mathrm{RI})^{34}$.

Outcomes. Disease remission and relapse were described as before ${ }^{21}$. Briefly, the initial 6 th -months of treatment was defined as the remission induction stage, and remission was defined as fulfilling each of the following after 6-month treatment: (1) $\geq 50 \%$ decline in the IgG4-RD RI; (2) GC tapered to maintenance dose (prednisone $\leq 10 \mathrm{mg} /$ day); and (3) no relapse during GC tapering (within 6 months). Clinical symptoms recurred, or imaging findings worsened with or without IgG4 level increased were considered as disease relapse in patients who had a follow-up period of 3 months or longer.

Statistical analysis. Continuous variables are described in mean and standard deviation (SD) or median and interquartile range (IQR) according to the data distribution. Comparisons between the two groups were performed using Student's $t$-test or the appropriate non-parametrical test, depending on normal or non-normal data distribution. Categorical variables were expressed as percentages, and $\chi^{2}$-test was used for comparisons. Fisher's exact test was used when one or more expected frequencies were less than 5 . Correlation between two continuous non-normal variables was analyzed using Spearman's rank correlation. Univariate logistic regression was used to predict risk factors for eosinophilia at 12 months follow-up. Statistical analysis was performed by SPSS version 24.0 and Graphpad Prism version 7.0. A $P$-value $<0.05$ was considered statistically significant.

Compliance with ethical standards. This study complied with the Declaration of Helsinki and was approved by the Human and Animal Ethics Review Committees of PUMCH, China (approval number: S-442). Informed consent was obtained from all individual participants included in the study.

\section{Data availability}

The datasets used and or analyzed during the current study are available from the corresponding author on reasonable request.

Received: 20 May 2019; Accepted: 22 October 2019;

Published online: 11 November 2019

\section{References}

1. Klion, A. D. Eosinophilia: a pragmatic approach to diagnosis and treatment. Hematology Am. Soc. Hematol. Educ. Program 2015, 92-97 (2015).

2. Rothenberg, M. E. \& Hogan, S. P. The eosinophil. Annu. Rev. Immunol. 24, 147-174 (2006).

3. Kovalszki, A. \& Weller, P. F. Eosinophilia. Prim Care. 43, 607-617 (2016).

4. Umehara, H. et al. Current approach to the diagnosis of IgG4-related disease - Combination of comprehensive diagnostic and organspecific criteria. Mod. Rheumatol. 27, 381-391 (2017).

5. Carruthers, M. N. et al. IgG4-related disease and lymphocyte-variant hypereosinophilic syndrome: A comparative case series. Eur. J. Haematol. 98, 378-387 (2017).

6. Saeki, T. et al. Comparison of clinical and laboratory features of patients with and without allergic conditions in IgG4-related disease: A single-center experience in Japan. Mod. Rheumatol. 2018, 1-4 (2018).

7. Culver, E. L. et al. Increases in IgE, eosinophils, and mast cells can be used in diagnosis and to predict relapse of IgG4-related disease. Clin. Gastroenterol. Hepatol. 15, 1444-1452 (2017).

8. Kuruma, S. et al. Allergen-specific IgE antibody serologic assays in patients with autoimmune pancreatitis. Intern. Med. 53, 541-543 (2014).

9. Kamisawa, T., Anjiki, H., Egawa, N. \& Kubota, N. Allergic manifestations in autoimmune pancreatitis. Eur. J. Gastroenterol. Hepatol. 21, 1136-1139 (2009).

10. Della-Torre, E. et al. Prevalence of atopy, eosinophilia, and IgE elevation in IgG4-related disease. Allergy 69, 269-272 (2014).

11. Sah, R. P. et al. Eosinophilia and allergic disorders in autoimmune pancreatitis. Am. J. Gastroenterol. 105, 2485-2491 (2010).

12. Chen, L. Y. et al. Polyclonal hyperviscosity syndrome in IgG4-related disease and associated conditions. Clin. Case Rep. 3, 217-226 (2015).

13. Chen, C., Chen, K., Huang, X., Wang, K. \& Qian, S. Concurrent eosinophilia and IgG4-related disease in a child: A case report and review of the literature. Exp. Ther. Med. 15, 2739-2748 (2018).

14. Horiuchi, K. et al. Marked eosinophilia as the first manifestation of sclerosing cholangitis. Intern. Med. 48, 1377-1382 (2009).

15. Deshpande, V. et al. Consensus statement on the pathology of IgG4-related disease. Mod. Pathol. 25, 1181-1192 (2012).

16. Deshpande, V., Khosroshahi, A., Nielsen, G. P., Hamilos, D. L. \& Stone, J. H. Eosinophilic angiocentric fibrosis is a form of IgG4related systemic disease. Am. J. Surg. Pathol. 35, 701-706 (2011).

17. Wallace, Z. S. et al. Clinical phenotypes of IgG4-related disease: an analysis of two international cross-sectional cohorts. Ann Rheum Dis 78, 406-412 (2019).

18. Wang, M. et al. Differences and similarities between IgG4-related disease with and without dacryoadenitis and sialoadenitis: clinical manifestations and treatment efficacy. Arthritis Res Ther. 21, 44 (2019).

19. Bochner, B. S. The eosinophil: For better or worse, in sickness and in health. Ann. Allergy Asthma Immunol. 121, 150-155 (2018). 
20. Wallace, Z. S. et al. Predictors of disease relapse in IgG4-related disease following rituximab. Rheumatology (Oxford). 55, 1000-8 (2016).

21. Wang, L. et al. Failure of remission induction by glucocorticoids alone or in combination with immunosuppressive agents in IgG4related disease: a prospective study of 215 patients. Arthritis Res. Ther. 20, 65 (2018).

22. Mahajan, V. S., Mattoo, H., Deshpande, V., Pillai, S. S. \& Stone, J. H. IgG4-related disease. Annu. Rev. Pathol. 9, 315-347 (2014).

23. Miyake, K. et al. Peripheral CD4+ T cells showing a Th2 phenotype in a patient with Mikulicz's disease associated with lymphadenopathy and pleural effusion. Mod. Rheumatol. 18, 86-90 (2008).

24. Tanaka, A. et al. Th2 and regulatory immune reactions contribute to IgG4 production and the initiation of Mikulicz disease. Arthritis Rheum. 64, 254-263 (2012).

25. Grados, A. et al. T Cell Polarization toward TH2/TFH2 and TH17/TFH17 in Patients with IgG4-Related Disease. Front Immunol. 8, 235 (2017).

26. Heeringa, J. J. et al. Expansion of blood IgG4(+) B, TH2, and regulatory T cells in patients with IgG4-related disease. J. Allergy Clin. Immunol. 141, 1831-1843 (2018).

27. Moriyama, M. \& Nakamura, S. Th1/Th2 Immune Balance and Other T Helper Subsets in IgG4-Related Disease. Curr. Top Microbiol. Immunol. 401, 75-83 (2017).

28. Shiokawa, M. et al. Pathogenicity of IgG in patients with IgG4-related disease. Gut 65, 1322-1332 (2016).

29. Cunningham, A. S. Eosinophil counts: age and sex differences. J. Pediatr. 87, 426-427 (1975).

30. Bittner, J. J., Halberg, F. \& Hamerston, O. Sex difference in eosinophil counts in tall blood of mature B1 mice. Science 125, 73 (1957).

31. Chen, L. Y. C., Mattman, A., Seidman, M. A. \& Carruthers, M. N. IgG4-related disease: what a hematologist needs to know. Haematologica. 104, 444-455 (2019).

32. Umehara, H. et al. Comprehensive diagnostic criteria for IgG4-related disease (IgG4-RD), 2011. Mod. Rheumatol. 22, 21-30 (2012).

33. Johansson, S. G. et al. A revised nomenclature for allergy. An EAACI position statement from the EAACI nomenclature task force. Allergy 56, 813-824 (2001).

34. Carruthers, M. N., Stone, J. H., Deshpande, V. \& Khosroshahi, A. Development of an IgG4-RD Responder Index. Int. J. Rheumatol. 2012, 259408 (2012).

\section{Acknowledgements}

This work was supported by National Natural Science Foundation of China (No. 81571587, 81771757), Natural Science Foundation of Beijing Municipality (No. 7172178), Chinese Academy of Medical Sciences Initiative for Innovative Medicine (2017-I2M-3-001), and The National Key Research and Development Program of China (No. 2016YFC0901500).

\section{Author contributions}

X.Z., W.Z. and Y.Z. analyzed the data and wrote the manuscript. P.P.Z., J.Q.L., Y.J.H., Y.Y.F., L.Y.P. and Q.S. collected the patient's data. All authors reviewed the manuscript.

\section{Competing interests}

The authors declare no competing interests.

\section{Additional information}

Correspondence and requests for materials should be addressed to W.Z. or Y.Z.

Reprints and permissions information is available at www.nature.com/reprints.

Publisher's note Springer Nature remains neutral with regard to jurisdictional claims in published maps and institutional affiliations.

Open Access This article is licensed under a Creative Commons Attribution 4.0 International License, which permits use, sharing, adaptation, distribution and reproduction in any medium or format, as long as you give appropriate credit to the original author(s) and the source, provide a link to the Creative Commons license, and indicate if changes were made. The images or other third party material in this article are included in the article's Creative Commons license, unless indicated otherwise in a credit line to the material. If material is not included in the article's Creative Commons license and your intended use is not permitted by statutory regulation or exceeds the permitted use, you will need to obtain permission directly from the copyright holder. To view a copy of this license, visit http://creativecommons.org/licenses/by/4.0/.

(C) The Author(s) 2019 